\title{
Swiss embrace complementary medicine
}

Complementary medicine is the preferred term in Europe for a wide range of alternative methods of healing that have little else in common except that they often fall outside the bounds of mainstream medicine. In Europe generally, but in Switzerland in particular, there seems to be a growing interest by doctors and the public alike in unconventional methods of healing, such as homeopathy, herbs, acupuncture, and Ayurvedic medicine (an ancient Indian system of holistic medicine).

The increase in popularity of complementary medicine in Switzerland during the past few years has been phenomenal. It is estimated that between 50 and 80 per cent of the population (figures vary by region) would prefer to be treated by these methods, although in practice fewer than half receive such treatments.

\section{Reasons for interest}

This growing interest in some of the more unconventional methods of healing has been attributed to a number of factors:

High-tech medicine has become impersonal, reducing patients to mere numbers.

Patients today have higher expectations of cure and are often not willing to accept that some diseases such as cancer and AIDS are still mostly incurable.

Doctors do not spend enough time listening to patients, and patients are often reluctant to ask questions.

Patients who seek alternative treatments usually have faith in these methods (some of these have an effect similar to the placebo effect without the risk of toxicity of 'modern' medicines). There is a common belief that complementary treatments have fewer adverse effects than modern medicines, and there is general ignorance about the unpredictable toxic effects of some herbal medicines.

\section{Into the mainstream}

A number of physicians in Switzerland are now trying to integrate some forms of complementary medicine into the mainstream. However, most treatments in complementary medicine are given by independent non-medical practitioners without direct supervision by physicians. And, although the licensing requirements can vary, they are generally well regulated.
About 25 per cent of Swiss doctors have also received training in the use of at least one unconventional method. And some doctors who do not themselves practice such methods are willing to refer their patients to those who do. The use of unconventional methods by Swiss doctors is not necessarily an endorsement of their scientific validity: it simply means that the methods used are considered to be safe and felt to be of some help to patients.

Accurate figures of the numbers of actual treatments performed using complementary methods are hard to come by. Although Swiss insurance companies generally support the use of complementary medicine and would like to provide reimbursement for such treatments, a recent legally binding directive from the Swiss Minister of Health, Ruth Dreyfuss, stipulated that reimbursement should be limited to those methods that have been proven scientifically. Even so, it is possible in Switzerland to buy additional health insurance to cover treatments in complementary medicine without requiring scientific proof of the validity of the method.

\section{New resources}

Although there is still much controversy concerning the merits and validity of complementary medicine, there are some signs that the medical profession in Switzerland is beginning to embrace the idea. The University Hospital in Zurich last year created a new position and appointed a professor of complementary medicine. In other Swiss universities, complementary medicine is also being taught by part-time lecturers. A position for a fulltime professor of complementary medicine has been approved at the University of Bern but has not been filled yet.

In addition, the Swiss National Fund, the government agency for supporting scientific research, allocated Sf5 million (about $\$ 4$ million) in 1993 for support of 12 research projects in the following areas:

hypnosis in the management of pain and allergic disorders

health policy rules and regulations regarding complementary medicine an economic analysis on the effects of offering complementary medicine as a health benefit reimbursable by health insurance companies

the motives behind patient choice in the areas of conventional or complementary medicine

use of homeopathy versus conventional medicines during pregnancy

study of the postulated mechanism of circulatory effects of acupuncture via the autonomic nervous system (This project has been completed and no such mechanism could be demonstrated.)

controlled study of the effect of acupuncture on bronchial asthma

the effect of acupuncture in the treatment of insomnia

an assessment of the improvement in quality of life of cancer patients using unconventional medical therapies

the manual trigger-point treatment of chronic lumbo-sacral pain

study of foot reflex zone massage.

For a small country like Switzerland (population 6 million), this level of research funding compares favourably with institutions such as the US National Institutes of Health, which in 1992 established an Office of Alternative Medicine and in 1993, 1994, and 1995 allocated $\$ 2, \$ 3.5$, and $\$ 5.4$ million, respectively, to these areas of research.

Switzerland has also proposed an initiative called the European COST (Cooperation Européenne de la Recherche Scientifique et Technique). The goal of the initiative is to establish a common scientific basis, if any, of unconventional medicines, to demonstrate the potential for and limitations of such medicines, and to harmonize regulations for their control.

Switzerland is also home to several multinational pharmaceutical companies - several of which have already expressed an interest in the area. For example, a number of herbs have pharmacologically active constituents from which new drugs can be developed. In addition, the European sales of known herbal over-thecounter products already exceeds an estimated $\$ 2$ billion a year, according to an article in the British Medical Joumal (309, 107-111 (1994)).

If the interest in complementary medicine continues to build, and some methods of treatment are proven to be useful, it may not be too long before complementary medicine becomes part of the mainstream and the term becomes obsolete.

KEWAL JAIN

Medical writer, Basel 